\title{
Lost in quantification...: The influence of different software packages on flow quantification measures
}

\author{
C. Rischpler, MD, ${ }^{\mathrm{a}}$ and S. G. Nekolla, $\mathrm{PhD}^{\mathrm{a}}$ \\ a Department of Nuclear Medicine, Klinikum rechts der Isar, Technical University of Munich, \\ Munich, Germany
}

Received Nov 22, 2017; accepted Nov 22, 2017

doi: 10.1007/s12350-017-1159-6

\section{See related article, pp. 1243-1253}

Over the last decades, non-invasive myocardial perfusion imaging has shown major improvements and has consolidated its role as a mainstay for the workup of coronary artery disease. ${ }^{1}$ While the most often applied technique for myocardial perfusion imaging is still single-photon emission tomography (SPECT) imaging, positron emission tomography (PET) for myocardial perfusion imaging has gained growing importance and acceptance in clinical routine. Reasons for that development are-besides the persisting intermittent shortage of Technetium-99m-technical advantages such as reliable tracer uptake quantification, higher count sensitivity, higher temporal and spatial resolution of PET. ${ }^{2}$ Furthermore, PET myocardial perfusion imaging gets along with a substantially lower radiation exposure to the patient due to different physical properties of PET radiotracers such as shorter half-life. ${ }^{3}$ Last not least, the probably most important advantage of PET myocardial perfusion imaging is the potential for absolute quantification of myocardial blood flow, a technique which has first been described in the $80 \mathrm{~s} .{ }^{4}$ The superior extraction fraction of PET perfusion tracers compared to Technetium-99m-labeled SPECT agents and the calculation of absolute measures of myocardial blood flow also are main reasons for the superior diagnostic accuracy of PET over SPECT. ${ }^{5-7}$

\footnotetext{
Reprint requests: C. Rischpler, MD, Department of Nuclear Medicine, Klinikum rechts der Isar, Technical University of Munich, Munich, Germany; rischpler@gmail.com

J Nucl Cardiol 2019;26:1255-7.

$1071-3581 / \$ 34.00$

Copyright (C) 2018 American Society of Nuclear Cardiology.
}

However, absolute quantification of myocardial blood flow is not trivial and there are many factors that may have major impact on the calculated results. ${ }^{8}$ As indicated before, the different available PET perfusion tracers also have different physical (half-life, positron range etc. of the respective radionuclide) and biochemical [extraction/retention fraction, excretion mechanism, accumulation (e.g., N-13 ammonia, Rubidium-82) vs diffusion (O-15 water) etc.] properties, which all have to be taken into account. Also, the protocol needs to be designed carefully: for example, the generation of an accurate input function, which is calculated from a small region of interest (ROI) in the left atrium, left ventricular cavity, or the aorta when the radiotracer passes the heart may be incorrectly assessed if the radiotracer is injected too fast (i.e., with a too high activity concentration) which may drive the scanner into saturation. ${ }^{9}$ The ROI location for the estimation of the input function per se may also have a substantial effect on blood flow results. ${ }^{10}$ Obviously, motion and subsequent misalignment between PET and CT data may cause erroneous estimation of the myocardial blood flow. ${ }^{11}$ It goes without saying that different scanners with different properties (crystal material, 2D vs. 3D scanners, timeof-flight availability etc.) may result in different estimations of myocardial blood flow. ${ }^{12}$ Another important factor that may have substantial impact on myocardial blood flow estimation is the choice of kinetic model, which has to be picked carefully and in dependence of different factors, e.g., the applied perfusion tracer. ${ }^{13}$ Another important factor (also on the post-processing side), which may have substantial effect on flow estimation, is the used software package. Different software packages implement different kinetic models, different corrections (e.g., spillover from blood to myocardium and vice versa) and require a different degree of user interaction. There are many software solutions for kinetic analysis of PET data in cardiac imaging on the 


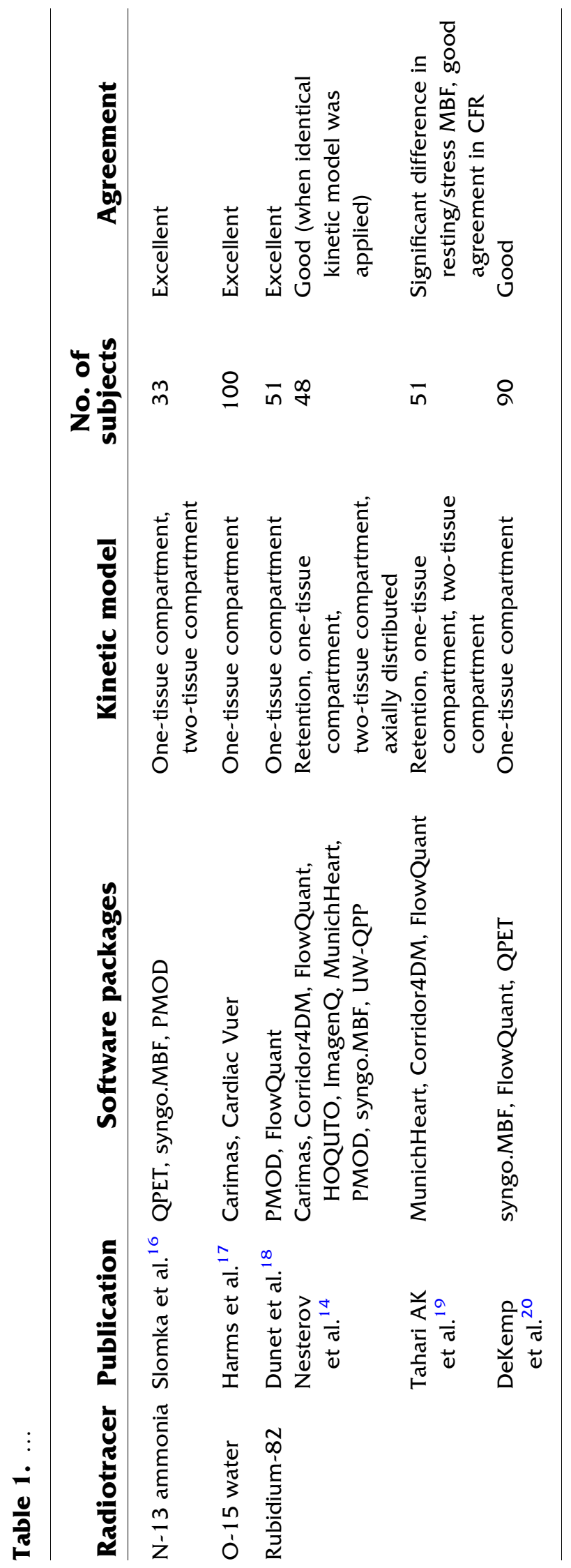

market. Often used software packages include "FlowQuant $^{\circledR}$, , (developed at the University of Ottawa Heart Institute, Canada), "Carimas" (developed at the University of Turku, Finland), "Cardiac VUer" (developed at the VU University Medical Center in Amsterdam, Netherlands), "HOQUTO" (developed Chietsugu Katoh and colleagues from the Hokkaido University, Japan), "Syngo MBF" (developed by Siemens Medical Solutions), "PMOD" (distributed by PMOD Technologies LLC, a spin-off of the Zurich PET center), "QPET" (developed by Cedars-Sinai, Los Angeles, California, USA) or "MunichHeart" (developed by one of the authors, Stephan Nekolla, at the Technical University Munich, Germany). Comparison of different software packages for the quantification of myocardial blood flow with different myocardial perfusion tracers have been performed in several studies (see Table 1). Most of these studies demonstrated a good to excellent agreement between the different software packages under the premise that the identical kinetic model was applied. For example, in one of the largest studies by Nesterov et al., a multicenter study comparing all existing software packages $(\mathrm{N}=10)$ for the quantification of Rb-82 PET myocardial perfusion imaging, the authors concluded that when using the identical kinetic model, software solutions might be interchangeable. ${ }^{14}$ While most such studies focused on the comparison of software packages in patients that were assessed for coronary artery disease in a daily clinical routine manner, the present manuscript focused on a certain subpopulation with structural changes of the left ventricular geometry, namely patients suffering from hypertrophic cardiomyopathy. This disease entity is the most common genetic cardiovascular disease in the general population and is therefore of daily importance in a cardiovascular imaging center. Patients with this disorder suffer from an increased left ventricular mass often with substantial hypertrophy of the septum, which is supplied by altered coronary arterioles and a reduced number of capillaries. It becomes obvious that these structural changes may result in erroneous segmentation of the left ventricular myocardial wall by the respective software and that also technical problems regarding e.g., partial volume and spillover correction may appear. Consequently, the authors of the present manuscript by Yalcin et al. found significant differences between the two tested software packages QPET and PMod in stress flow and coronary flow reserve values in patients with hypertrophic cardiomyopathy and high spillover fractions. The authors concluded that the anatomic characteristics in this patient population contribute to the differences found and that software packages may deliver substantial different myocardial blood flow values. Interestingly, the applied software 
packages ran the identical 2-tissue-compartment model but different computational implementations. One software solution, namely PMod, requires manual adjustment in the process of left ventricular wall segmentation, which raises the suspicion that this fact causes these differences in myocardial blood flow estimation. Unfortunately, as we have no gold standard in this study, the question what software delivers the correct myocardial blood flow estimations remains unanswered-but most likely, the software differed in their technical implementation, e.g., whether and how partial volume and spillover corrections as suggested for cardiac flow quantification already many years ago were applied. ${ }^{15}$ The absence or presence of motion correction is another factor which is crucial in the setting of scans which last for several minutes-especially in a post stress setting. This should all remind us that quantification software is not characterized by a single component—such as the choice of the particular kinetic model. The actual implementation is a rather multifactorial system, which might lead to differences between software packages based on the investigated patient cohort - a factor not commonly taken into consideration in studies comparing software.

Thus, the strength of this study is that it demonstrates that we have to act with high caution regarding myocardial blood flow quantification in patients with structural changes of the left ventricular wall and that further studies are warranted to elucidate the question what the best approach is to correctly quantify myocardial blood flow in such patient populations.

\section{Disclosure}

None.

\section{References}

1. Beller GA, Bergmann SR. Myocardial perfusion imaging agents: SPECT and PET. J Nucl Cardiol 2004;11:71-86.

2. Higuchi T, Bengel FM. Cardiovascular nuclear imaging: from perfusion to molecular function: Non-invasive imaging. Heart 2008;94:809-16

3. Gaemperli O, Bengel FM, Kaufmann PA. Cardiac hybrid imaging. Eur Heart J 2011;32:2100-8.

4. Bergmann SR, Fox KA, Rand AL, et al. Quantification of regional myocardial blood flow in vivo with $\mathrm{H} 215 \mathrm{O}$. Circulation 1984;70:724-33.

5. Bengel FM, Higuchi T, Javadi MS, Lautamaki R. Cardiac positron emission tomography. J Am Coll Cardiol 2009;54:1-15.
6. Bateman TM, Heller GV, McGhie AI, et al. Diagnostic accuracy of rest/stress ECG-gated Rb-82 myocardial perfusion PET: Comparison with ECG-gated Tc-99m sestamibi SPECT. J Nucl Cardiol 2006;13:24-33.

7. Flotats A, Bravo PE, Fukushima K, Chaudhry MA, Merrill J, Bengel FM. (8)(2)Rb PET myocardial perfusion imaging is superior to (9)(9)mTc-labelled agent SPECT in patients with known or suspected coronary artery disease. Eur J Nucl Med Mol Imaging 2012;39:1233-9.

8. Klein R, Beanlands RS, deKemp RA. Quantification of myocardial blood flow and flow reserve: Technical aspects. J Nucl Cardiol 2010;17:555-70.

9. Tout D, Tonge CM, Muthu S, Arumugam P. Assessment of a protocol for routine simultaneous myocardial blood flow measurement and standard myocardial perfusion imaging with rubidium- 82 on a high count rate positron emission tomography system. Nucl Med Commun 2012;33:1202-11.

10. Vasquez AF, Johnson NP, Gould KL. Variation in quantitative myocardial perfusion due to arterial input selection. JACC Cardiovasc Imaging 2013;6:559-68.

11. Lautamaki R, Brown TL, Merrill J, Bengel FM. CT-based attenuation correction in (82)Rb-myocardial perfusion PET-CT: Incidence of misalignment and effect on regional tracer distribution. Eur J Nucl Med Mol Imaging 2008;35:305-10.

12. Renaud JM, Yip K, Guimond J, et al. Characterization of 3-dimensional PET Systems for accurate quantification of myocardial blood flow. J Nucl Med 2017;58:103-9.

13. Choi Y, Huang SC, Hawkins RA, et al. Quantification of myocardial blood flow using 13N-ammonia and PET: Comparison of tracer models. J Nucl Med 1999;40:1045-55.

14. Nesterov SV, Deshayes E, Sciagra R, et al. Quantification of myocardial blood flow in absolute terms using (82)Rb PET imaging: The RUBY-10 study. JACC Cardiovasc Imaging 2014;7:1119-27.

15. Beanlands RS, Muzik O, Hutchins GD, Wolfe ER Jr, Schwaiger M. Heterogeneity of regional nitrogen 13-labeled ammonia tracer distribution in the normal human heart: Comparison with rubidium 82 and copper 62-labeled PTSM. J Nucl Cardiol 1994;1:225-35.

16. Slomka PJ, Alexanderson E, Jacome R, et al. Comparison of clinical tools for measurements of regional stress and rest myocardial blood flow assessed with $13 \mathrm{~N}$-ammonia PET/CT. J Nucl Med 2012;53:171-81.

17. Harms HJ, Nesterov SV, Han C, et al. Comparison of clinical noncommercial tools for automated quantification of myocardial blood flow using oxygen-15-labelled water PET/CT. Eur Heart J Cardiovasc Imaging 2014;15:431-41.

18. Dunet V, Klein R, Allenbach G, Renaud J, deKemp RA, Prior JO. Myocardial blood flow quantification by Rb-82 cardiac PET/CT: A detailed reproducibility study between two semi-automatic analysis programs. J Nucl Cardiol 2016;23:499-510.

19. Tahari AK, Lee A, Rajaram M, et al. Absolute myocardial flow quantification with (82)Rb PET/CT: Comparison of different software packages and methods. Eur J Nucl Med Mol Imaging 2014;41:126-35.

20. Dekemp RA, Declerck J, Klein R, et al. Multisoftware reproducibility study of stress and rest myocardial blood flow assessed with 3D dynamic PET/CT and a 1-tissue-compartment model of 82Rb kinetics. J Nucl Med 2013;54:571-7. 